\title{
A simple method to detect plant based inhibitors of glycation induced protein cross-linking
}

\author{
H.K.I. Perera1, H.A.S.K. Ranasinghe ${ }^{1,2}$ \\ ${ }^{1}$ Department of Biochemistry, Faculty of Medicine, University of Peradeniya, Sri Lanka, ${ }^{2}$ Postgraduate Institute of Science, \\ University of Peradeniya, Sri Lanka
}

\section{A B S T R A C T}

Background: Glycation induced cross-linking of proteins are associated with chronic diabetic complications. Inhibition of protein glycation is one of the therapeutic approaches to prevent the progression of diabetic complications. Objective: Objective of this study was to establish a simple method to identify medicinal plants which can inhibit glycation induced protein cross-linking. Methods: Lysozyme was incubated at $37^{\circ} \mathrm{C}$ up to 4 weeks with different concentrations of glucose, fructose and ribose in sodium phosphate buffer (pH 7.4). Appropriate controls and blanks were carried out. Aminoguanidine (AG) was used as the standard inhibitor. Water extracts of Bryophyllum pinnatum leaves, Coriandrum sativum seed and Murraya koenigi leaves were used as potential inhibitors. Aliquots were removed from the incubation mixtures at intervals and analyzed for the presence of $A G E$ induced protein cross-links, using SDS-PAGE. Appearance and the intensity of high molecular weight products were compared. Results: Extent of cross-linking was dependent on the sugar concentration. Cross-linking was slowest in the presence of glucose and fastest in the presence of ribose. AG inhibited glycation induced protein cross-linking in the presence of all three sugars. B. pinnatum leaves, $C$. sativum seeds and $M$. koenigi leaves inhibited protein cross-linking in the presence of sugar. This inhibition was greater than that of AG. Conclusions: We have established a simple SDS-PAGE method to identify medicinal plants which inhibit glycation induced protein cross-linking. We also demonstrated the effectiveness of $B$. pinnatum leaves, $C$. sativum seed and $M$. koenigi leaves in inhibiting glycation induced protein cross-linking in vitro.

Key words: Glycation, Protein cross-links, SDS-PAGE, Bryophyllum pinnatum, Coriandrum sativum and Murraya koenigi

\section{INTRODUCTION}

Protein glycation plays a key role in the development of chronic complications associated with diabetes mellitus such as atherosclerosis, nephropathy retinopathy and neuropathy. ${ }^{1-4}$ Protein glycation is initiated as a non enzymatic reaction between reducing sugars and proteins followed by a series of reactions leading to formation of advanced glycation end products (AGE). AGE formation occurs mainly on long-lived proteins such as lens crystallins and collagen. ${ }^{4}$

During later events of glycation, some AGEs form inter or intra molecular cross-links leading to severe structural and functional changes especially protein/protein and protein/cell interactions in the vascular wall. Cross-linking of extracellular matrix proteins such as collagen and elastin, leads to increased vascular stiffness and diminished arterial and myocardial compliance leading to organ damage. ${ }^{3}$ Cross-linking of collagen in the kidney is thought to be directly responsible for the advancement of impaired renal function. ${ }^{5}$ In the eye lens, AGEs induce structural destabilization of the proteins causing conformational changes leading to the formation of protein aggregates that scatter the light. ${ }^{4}$

Current scientific literature reveals that the inhibition of AGE formation is one of the therapeutic approaches to 
prevent the progression of diabetic complications. Many traditional medicinal plants have being used widely for the treatment of diabetes and diabetic complications since ancient times. ${ }^{6}$ In this regard, efforts have been directed in validating medicinal plants with protein glycation inhibitory potential. ${ }^{7,8}$ However, adequate work has not yet been done in Sri Lanka on this area. Analytical techniques available to identify protein glycation inhibitors require expensive specialized equipment. Here we established a simple, cost effective technique to fulfill such drawbacks. Objective of this study was to establish a simple method to identify medicinal plants which can inhibit glycation induced protein cross-linking.

\section{MATERIALS AND METHODS}

We have adapted the method of Muthenna et al., with modifications to detect the formation of glycation-induced high molecular weight products. ${ }^{4}$ Lysozyme from chicken egg white (Sigma-Aldrich, USA) was incubated at $37^{\circ} \mathrm{C}$ up to 4 weeks with different concentrations $(0,100,250$ or 300 and $500 \mathrm{mM}$ ) of D-glucose, D-fructose and D-ribose in sodium phosphate buffer ( $\mathrm{pH} 7.4$ ) containing $0.02 \%$ sodium azide. Incubations were carried out in sealed tubes. Appropriate controls and blanks were carried out. Aminoguanidine (AG) $(10 \mathrm{mM})$ was used as the standard inhibitor.

Plant extracts that showed inhibition of protein glycation as observed using a novel method conducted by us previously, were used as potential inhibitors of protein cross-linking. Specimens of Bryophyllum pinnatum (Lam.) Oken [Synonym Kalanchoe pinnata (Lam.) Pers.] (Akkapana in Sinhalese) and Murraya koenigii (L.) Spreng (Curry leaf) were authenticated at the National herbarium, Royal Botanical Gardens, Peradeniya, Sri Lanka. Coriandrum sativum (Coriander) seeds were purchased as a branded product from open market. Water extracts $(5 \mathrm{mg} / \mathrm{ml})$ of three plant parts were prepared. Fructose and glucose were used in the incubations with plant extracts.

Aliquots were removed from the incubation tubes at intervals and stored at $-40^{\circ} \mathrm{C}$ until further analysis. AGE induced cross-linking of proteins present in the aliquots was detected using sodium dodecyl sulphate polyacrylamide gel electrophoresis (SDS-PAGE). Broad range molecular weight markers (Promega) were used to assess the approximate molecular weight of the high molecular weight products. Samples collected from incubations were loaded to the gel after heating with SDS sample buffer (2:1) at $95^{\circ} \mathrm{C}$. Electrophoresis was conducted using $12 \%$ gels (Enduro Vertical Gel Electrophoresis system- E2010-P) with a constant current of $30 \mathrm{~mA}$ per gel for about 90 min according to the standard Laemmli method. ${ }^{10}$
After separation at $\mathrm{pH}$ 8.6, protein bands were visualized by staining with Coomassie brilliant blue for $30 \mathrm{~min}$ and destained overnight. Appearance and the intensity of high molecular weight products were compared.

\section{RESULTS}

High molecular weight products of protein were formed when lysozyme was incubated in the presence of sugar as a result of glycation induced protein cross-linking (Figures 1-4). They represented the MW of dimer, trimer and tetramer of lysozyme as compared with the molecular weight markers (loaded with most of the gels). Extent of cross-linking was dependent on the sugar concentrations used, up to a certain limit. This feature was observed with fructose at the concentrations used for the assay (Figures 1 and 2). Cross-linking was fastest in the presence of ribose (Figures 1-3). Ribose promoted cross-linking at much lower concentrations and showed similar effects at both 100 and $500 \mathrm{mM}$ (Figure 1). Cross-linking was slowest in the presence of glucose (Figures 2-4) and the effect was not prominent enough to see the differences between different concentrations of glucose (Figure 2). Glucose showed only the dimer formation under the experimental conditions used in our study (Figures 2-4). Concentrations of high molecular weight products were increased with longer incubations (Figures 2-4). AG inhibited glycation induced protein cross-linking in presence of all three sugars (Figures 3 and 4).

B. pinnatum leaves, C. sativum seed and M. koenigi leaves inhibited protein cross-linking in the presence of both glucose and fructose. This inhibition was greater than that of AG (Figure 4). However the inhibitory effects on protein cross-

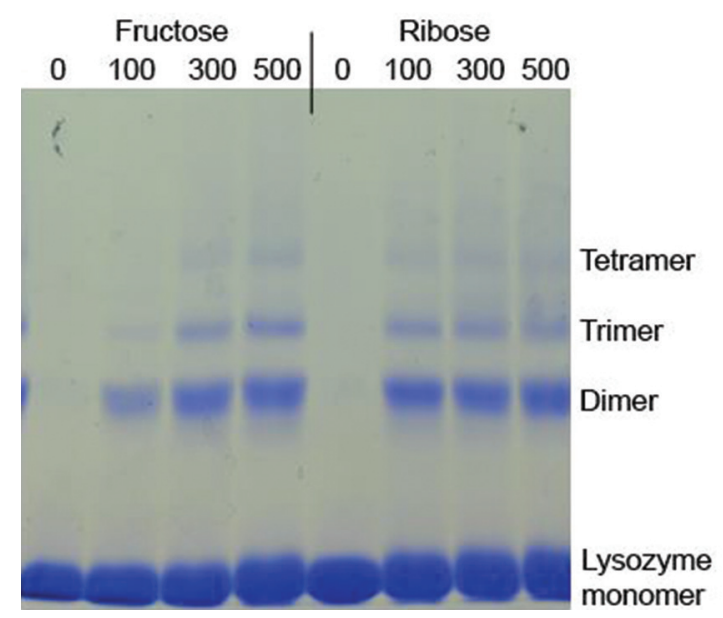

Figure 1: Effect of concentration of fructose and ribose on glycation induced protein cross-linking. SDS-PAGE was conducted using $12 \%$ gels. Lysozyme was incubated with fructose or ribose at concentrations of $0,100,300$ and $500 \mathrm{mM}$ for 10 days. High molecular weight products formed as a result of lysozyme cross-linking are indicated 


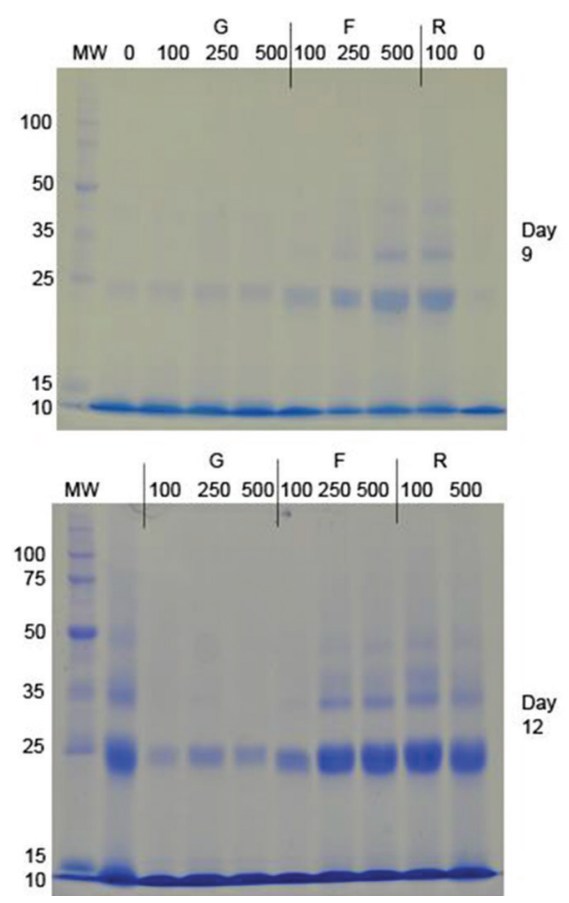

Figure 2: Effect of different concentrations of sugars on lysozyme cross-linking. SDS-PAGE was conducted using $12 \%$ gels. Lysozyme was incubated with glucose or fructose or ribose at concentrations of $0,100,250$ and $500 \mathrm{mM}$. Incubation periods were 9 (top) and 12 (bottom) days. G: incubation with glucose, F: incubation with fructose, R: incubation with ribose, MW: Molecular weight markers

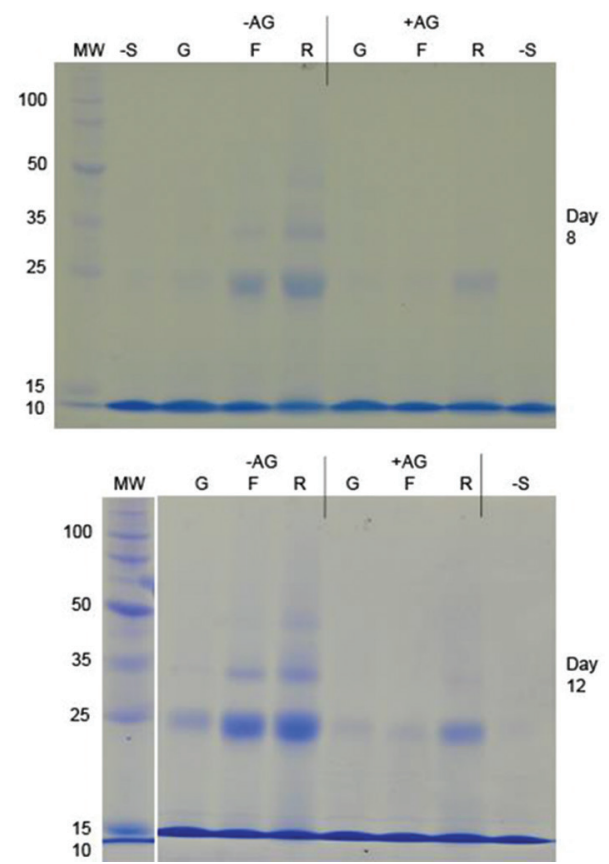

Figure 3: Effect of $A G$ in the presence of different sugars on lysozyme cross-linking. SDS-PAGE was conducted using $12 \%$ gels. Lysozyme was incubated with or without sugar in the presence or absence of AG. Incubation periods were 8 (top) and 12 (bottom) days. Concentration of the sugars used was $250 \mathrm{mM}$. -S: incubation without sugar, $\mathrm{G}$ : incubation with glucose, $\mathrm{F}$ : incubation with fructose, R: incubation with ribose, +AG: incubation with aminoguanidine, -AG: incubation without aminoguanidine, MW: Molecular weight markers

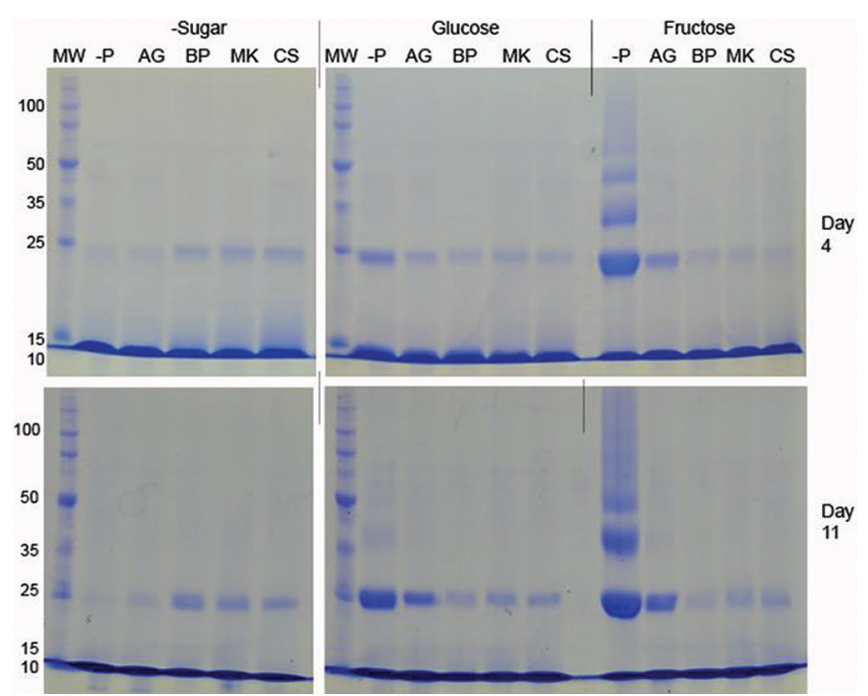

Figure 4: Effect of plant extracts on lysozyme cross-linking with or without glucose and fructose. SDS-PAGE was conducted using $12 \%$ gels. Lysozyme was incubated with or without sugar $(500 \mathrm{mM}$ glucose or $250 \mathrm{mM}$ fructose) in the presence or absence of plant extracts. Incubation periods were 4 (top) and 11 (bottom) days. Glucose: incubation with glucose, Fructose: incubation with fructose, - Sugar: incubation without sugar, MW: Molecular weight markers, -P: incubation without a plant extract, BP: incubation with Bryophyllum pinnatum, CS: incubation with Coriandrum sativum, MK: incubation with Murraya koenigi, AG: incubation with aminoguanidine

linking were more clearly seen in the presence of fructose. Inhibitory effect of plant extracts and $A G$ were evident even during early periods of incubation such as day 4 (Figure 4).

\section{DISCUSSION}

Analytical techniques available to identify protein glycation inhibitors require expensive methods such as high performance liquid chromatography, mass spectrometry, fluorescence spectrometry and specific ELISA assays. ${ }^{11-14}$ We have developed a simple method which can be conducted without sophisticated equipments, to identify plant based inhibitors of glycation induced protein crosslinking. SDS-PAGE method was conducted previously to detect inhibitory effects of pure compounds on lysozyme cross-linking. ${ }^{4,15,16}$ However, according to our knowledge, this is the first time reporting the suitability of this technique in detecting inhibitory potential of crude extracts of medicinal plants in glycation induced lysozyme cross-linking.

Lysozyme was used as a model protein previously to show glycation associated protein cross-linking., ${ }^{415-17}$ Lysozyme should serve as a good candidate for glycation as a protein with a $\mathrm{pI}$ of 11.35 and having 6 Lysine residues and 11 Arginine residues ${ }^{18}$ which are the targets of glycation. Previously, SDS-PAGE was used at various gel percentages $(12,15$ and $20 \%)$ to study the effect of glucose $(100$, 
$200 \mathrm{mM})$, fructose $(100 \mathrm{mM})$ and ribose $(100,250$, $400 \mathrm{mM}$ ) on lysozyme cross-linking, to study the effect of AG $(10 \mathrm{mM})$ and to study the effects of pure compounds on lysozyme cross-linking., ${ }^{415-17}$ In one study a SDS-gradient PAGE was used..$^{15}$ In these studies, incubations have been carried out at $\mathrm{pH} 7.4$ and $37^{\circ} \mathrm{C}$ for 1 week in most cases and 3 or 4 weeks in other studies. In some studies penicillin and streptomycin have been used during the incubations in addition to sodium azide.

Among the three sugars used in our study, results with D-ribose clearly demonstrated that the extent of crosslinking increased more rapidly with ribose. Several previous studies also have used ribose. In our study, glucose showed the slowest rate of glycation compared to other two sugars even at $500 \mathrm{mM}$ concentration. Even though glucose is the principle sugar found in the biological systems, our results suggest that, for in vitro experiments glucose is not ideal as the glycation reaction is too slow to detect the changes in the concentration of glycation products within a relatively shorter period. On the other hand, ribose was too strong in its glycation potential which may mask the effects of potential inhibitors. Rapid effects of ribose and slower effects of glucose on protein glycation were observed previously too, using different approaches. ${ }^{9,13}$ We propose that fructose is better compared to glucose and ribose, in detecting glycation induced cross-link formation as its effects occur at a more manageable rate.

Sakai et al., showed that fructose promote glycation induced lysozyme cross-linking at a faster rate compared to glucose, using a SDS-gradient PAGE. ${ }^{15}$ They used $100 \mathrm{mM}$ sugar and an incubation period of 4 weeks. They also showed the inhibition of cross-linking in presence of $10 \mathrm{mM}$ AG. Findings of Sakai et al., were similar to the findings of our study conducted using a standard mini gel. ${ }^{15}$ These findings suggest a more rapid effect of fructose in the development of diabetic complications.

Ellagic acid a flavanoid present in plants inhibited cross-linking of lysozyme incubated for 1 week in presence of $400 \mathrm{mM}$ ribose. Ellagic acid showed a dose-dependent inhibition of lysozyme cross-linking as monitored by $12 \%$ SDS-PAGE. ${ }^{4}$

Li et al., demonstrated some inhibitory effects of two compounds, 4-O-demethylsilvaticol and (-)-mitorubrin isolated from the fungus Paecilomyces sp. on lysozyme crosslinking, using 15\% SDS-PAGE. ${ }^{19}$ Those incubations were carried out in the presence of $100 \mathrm{mM}$ ribose for 7 days.

The effect of TRC4149 a synthetic AGE inhibitor on lysozyme cross-linking in the presence of $250 \mathrm{mM}$ ribose for 3 weeks was evaluated by SDS-PAGE. Treatment with TRC4149 reduced the formation of cross-linked proteins. ${ }^{16}$
Rahbar and Lalezari used lysozyme incubated with glucose $(200 \mathrm{mM})$ or fructose $(100 \mathrm{mM})$ to evaluate the inhibitory effect of test compounds (derivatives of aryl and heterocyclic ureido and aryl and heterocyclic carboxamido phenoxy isobutyric acids) at $1 \mathrm{mM}$ concentration, on AGEderived cross-linking. ${ }^{17}$ Incubation mixtures were analyzed after 7 days, using 20\% SDS-PAGE gels.

Our results obtained with a comparatively lower percentage of gel $(12 \%)$ gave more prominence to the high molecular weight products formed as a result of cross-linking, where as in studies conducted with higher gel percentages showed prominent bands with the native monomeric protein. Hence, we propose that $12 \%$ gel is more suitable for this procedure, as the focus is on the cross-linked high molecular products.

Saraswat et al., analyzed several dietary agents on their inhibitory effects on protein glycation. ${ }^{20}$ They used an eye lens soluble protein as model protein and fructose $(100 \mathrm{mM})$ as model sugar for in vitro glycation. Seventeen dietary materials at various concentrations of the extracts $(0 \cdot 01,0 \cdot 1,1 \cdot 0$ and $10 \mathrm{mg} / \mathrm{ml})$ were used. Reaction tubes were incubated for 3 weeks. They monitored protein crosslink formation using on SDS-PAGE and quantified the extent of cross-linking by measuring the density of relevant bands. Effects on preventing protein cross-links were observed with the aqueous extracts of ginger, cinnamon, cumin, green tea, lemon, apple, garlic and black pepper showing inhibitory effects of $30-80 \%$ at the concentration of $1 \mathrm{mg} / \mathrm{ml}^{20}$ (The images of the gels were not included in the paper).

Our results clearly demonstrated the inhibitory potential of B. pinnatum leaves, C. sativum seed and M. koenigi leaves on the formation of glycation induced cross-linking. We have previously established a novel electrophoresis method conducted under native conditions to detect protein glycation inhibitors using bovine serum albumin. ${ }^{9}$ The three plant extracts used in the present study were selected based on their protein glycation inhibitory effects observed previously with that novel method. Even though we have used an incubation period up to 4 weeks during optimization, an incubation of 1 week was sufficient to detect the inhibitory potential of plant extracts and the standard inhibitor on protein cross-linking.

C. sativum and M. koenigii are used as natural flavoring agents during cooking and for their medicinal effects. Administration of $M$. koenigii leaf extract to strpotozotocininduced diabetic rats showed a decline in $\mathrm{HbA}_{1 \mathrm{C}}$ level and a protective role against diabetic neuropathy. ${ }^{21}$ Free radical scavenging activity, antioxidant activity and nephroprotective effects of $M$. koenigii leaf are 
also proven. ${ }^{22}$ In vitro antiglycation activities of $50 \%$ ethanol extracts of $C$. sativum seed and $M$. koenigi were demonstrated previously using spectrofluorimetry with bovine serum albumin incubated with glucose $(200 \mathrm{mM})$ for 2 to 12 weeks. ${ }^{23} \mathrm{HbA}_{1 \mathrm{C}}$ lowering effect of B. pinnatum leaves in streptozotocin-induced diabetic rats was shown in another study. ${ }^{24}$ Our results provide further evidence on antiglycation properties of the three plants investigated, which could even inhibit protein cross-linking.

Hypoglycaemic effects of B. pinnatum leaves, ${ }^{24,25}$ C. sativum ${ }^{26}$ and $M$. koenigi $2^{2,27}$ were shown using streptozotocin-induced diabetic rats previously. C. sativum extract also showed increased insulin release from the $\beta$-cells of the pancreas in streptozotocin-induced diabetic rats. ${ }^{26}$ The antiglycating effects observed in the present study were independent of their known hypoglycaemic effects, as the sugar concentrations were similar in presence and absence of the plant extracts.

The technique established in our study can be used to observe protein cross-linking and the effect of medicinal plants in inhibiting protein cross-linking. However, in the SDS-PAGE method, though we can detect inhibition of protein cross-linking, the stage at which this inhibition was taking place (early or latter) was not identified. Our results are important to validate the glycation induced protein cross-linking inhibitory potential of medicinal plants to identify suitable candidates which can reduce diabetic complications in the future.

\section{CONCLUSION}

We have established a simple SDS-PAGE method to identify plant based inhibitors which can delay or prevent glycation induced protein cross-linking. Further, we have demonstrated the effectiveness of Bryophyllum pinnatum leaves, Coriandrum sativum seed and Murraya koenigi leaves in inhibiting glycation induced protein cross-linking in vitro. This inhibition was greater than that of $10 \mathrm{mM} \mathrm{AG}$.

\section{ACKNOWLEDGEMENTS}

Financial assistance from the Peradeniya University Research grant RG/AF/2013/33/M. Prof. R. Sivakanesan for revising the manuscript and Mr. A.M.P.S.T.M Bandara for technical assistance.

\section{REFERENCES}

1. Jakus $\mathrm{V}$ and Rietbrock N. Advanced glycation end-products and the progress of diabetic vascular complications Physiol Res 2004; 53: 131-142.

2. Nawale RB, Mourya VK and Bhise SB. Non enzymatic glycation of proteins: A cause for complication of Diabetes. Indian Journal of Biochemistry and Biophysics 2006; 43: 337-344.

3. Goh SY and Cooper ME. The role of advanced glycation end products in progression and complications of diabetes. Journal of Clinical Endocrinology and Metabolism 2008; 93(4): 1143-1152.

4. Muthenna P, Akileshwari C and Reddy GB. Ellagic acid, a new antiglycating agent: its inhibition of $\mathrm{N} \varepsilon$-(carboxymethyl) lysine. Biochem. J 2012; 442: 221-230.

5. Forbes JM, Cooper ME, Oldfield MD and Thomas MC. Role of advanced glycation end products in diabetic nephropathy. Journal of the American Society of Nephrology 2003; 14(3): 254-258.

6. Grover JK, Yadav S, and Vitas V. Medicinal plants of India with antidiabetic potential. J Ethnopharmacology 2002; 81: 81-100.

7. Wu C, Huang S, Lin $\mathrm{J}$ and Yen $\mathrm{G}$. Inhibition of advanced glycation endproduct formation by foodstuffs. Food Funct 2011; 2: 224-234.

8. Odjakova M, Popova E, Al Sharif M and Mironova R. Plantderived agents with anti-glycation activity 2012; http://dx.doi. org/10.5772/48186.

9. Wijetunge DCR and Perera HKI. A novel in vitro method to identify protein glycation inhibitors. Asian Journal of Medical Science 2014; 5(3): 15-21.

10. Electrophoresis Technical Handbook: [online]2010. Available from: URL: http://sinapseinc.com/files/Handbook-Eletroforese.pdf

11. Povichit N, Phrutivorapongkul A, Suttajit M, Chaiyasut $C$ and Leelapornpisid P. Phenolic content and in vitro inhibitory effects on oxidation and protein glycation of some Thai medicinal plants. Pak J Pharm Sci 2010; 23(4): 403-408.

12. Chen Y-F, Roan H-Y, Lii C-K, Huang Y-C and Wang T-S. Relationship between antioxidant and antiglycation ability of saponins, polyphenols, and polysaccharides in Chinese herbal medicines used to treat diabetes J Medicinal Plants Research 2011; 5(11): 2322-2331.

13. Wei $Y$, Chen L, Chen J, Ge L and He RQ. Rapid glycation with D-ribose induces globular amyloid-like aggregations of BSA with high cytotoxicity to SH-SY5Y cells. BMC Cell Biology 2009; 10:10 doi: 10.1186/1471-2121-10-10.

14. Ayatollahi SAM, Kobarfard F, Asgarpanah $\mathrm{J}$ and Choudhary MI. Antiglycation activity of Otostegia persica (Burm.) Boiss. Afr $J$ Biotechnology 2010; 9(24): 3645-3648.

15. Sakai M, Oimomi $M$ and Kasuga M. Experimental studies on the role of fructose in the development of diabetic complications. Kobe Journal of Medical Sciences 2002; 48(5/6): 125-136.

16. Pathak P, Gupta R, Chaudhari A, Shiwalkar A, Dubey A, Mandhare AB, et al. A novel advanced glycation end product breaker TRC4149 improves hemodynamic status in diabetic spontaneously hypertensive rats. Eur J Med Res 2008; 13: 388-398.

17. Rahbar S and Lalezari I. U.S. Patent No. 6,337,350. 2002; Washington, DC: U.S. Patent and Trademark Office.

18. Tagami U, Akashi S, Mizukoshi T, Suzuki E and Hirayama K. Structural studies of the Maillard reaction products of a protein using ion trap mass spectrometry. J. Mass Spectrom 2000; 35: 131-138.

19. Li D, Shigetomi K, Mitsuhashi S and Ubukata M. Maillard reaction inhibitors produced by Paecilomyces sp. Bioscience, biotechnology, and biochemistry 2013; 77(12): 2499-2501.

20. Saraswat M, Reddy PY, Muthenna P and Reddy GB. Prevention of non-enzymic glycation of proteins by dietary agents: prospects for alleviating diabetic complications. British Journal of Nutrition 2009; 101: 1714-1721.

21. Tembhurne SV and Sakarkar DM. Influence of Murraya koenigii on experimental model of diabetes and progression of neuropathic pain. Res Pharm Sci 2010; 5(1): 41-47. 
22. Gupta P, Nahata A and Dixit VK. An update of Murraya koenigii Spreng: a multifunctional Ayurvedic herb. J Chinese Integrative Medicine 2011; 9(8): 824-833.

23. Ramkissoon JS, Mahomoodally MF, Ahmed N and Subratty AH. Relationship between total phenolic content, antioxidant potential, and antiglycation abilities of common culinary herbs and spices. Journal of medicinal food 2012; 15(12): 11161123.

24. Patil SB, Dongare VR, Kulkarni CR, Joglekar MM and Arvindekar AU. Antidiabetic activity of Kalanchoe pinnata in streptozotocin-induced diabetic rats by glucose independent insulin secretagogue action. Pharm Biol 2013; 51(11):14111418.
25. Ogbonnia SO, Odimegwu $\mathrm{J}$ and Enwuru V. Evaluation of hypoglycaemic and hypolipidaemic effects of aqueous ethanolic extracts of Treculia africana Decne and Bryophyllum pinnatum Lam. and their mixture on streptozotocin (STZ)-induced diabetic rats. African Journal of Biotechnology 2008; 7 (15): 2535-2539.

26. Eidi M, Eidi A, Saeidi A, Molanaei S, Sadeghipour A, Bahar M et al. Effect of coriander seed (Coriandrum sativum L.) ethanol extract on insulin release from pancreatic beta cells in streptozotocininduced diabetic rats. Phytother Res. 2009; 23(3):404-406.

27. Dineshkumar B, Mitra A and Mahadevappa M. Antidiabetic and hypolipidemic effects of mahanimbine (carbazole alkaloid) from Murraya koenigii (rutaceae) leaves. International Journal of Phytomedicine 2010; 2(1): 22-30.

\section{Authors Contribution:}

HKIP - Concept and design of the study, literature search, obtaining grants, data collection, analysis and interpretation, manuscript preparation and critical revision of the manuscript; HASKR - Literature search, conducting experiments and data collection.

Source of Support: Peradeniya University Research grant RG/AF/2013/33/M, Conflict of Interest: None declared. 